\title{
Prognostic Utility of the Combination of Platelet Count with Neutrophil-to-Lymphocyte Ratio in Aged Patients with Acute Myocardial Infarction Undergoing Percutaneous Coronary Intervention
}

\author{
Xiao-Qing Quan $\mathbb{D}^{1},{ }^{1}$ Hong-Yan Ji $\mathbb{D},{ }^{2}$ Jie Jiang $\mathbb{D},{ }^{2}$ Jia-Bao Huang $\mathbb{D},{ }^{3}$ \\ and Cun-Tai Zhang ${ }^{4}$ \\ ${ }^{1}$ Department of General Practice, Shenzhen Longhua District Central Hospital, \\ The Affiliated Central Hospital of Shenzhen Longhua District, Guangdong Medical University, Shenzhen, China \\ ${ }^{2}$ Second Clinical Medical College, Tongji Medical College, Huazhong University of Science and Technology, Wuhan, China \\ ${ }^{3}$ Department of General Practice, The Third Affiliated Hospital of Sun Yat-sen University, Guangzhou, Guangdong, China \\ ${ }^{4}$ Department of Geriatrics, Tongji Hospital, Tongji Medical College, Huazhong University of Science and Technology, \\ Wuhan, China
}

Correspondence should be addressed to Jia-Bao Huang; 15972990502@163.com and Cun-Tai Zhang; ctzhang@tjh.tjmu.edu.cn

Received 12 January 2020; Revised 30 March 2021; Accepted 6 April 2021; Published 24 April 2021

Academic Editor: Canan Akman

Copyright (C) 2021 Xiao-Qing Quan et al. This is an open access article distributed under the Creative Commons Attribution License, which permits unrestricted use, distribution, and reproduction in any medium, provided the original work is properly cited.

\begin{abstract}
Background and Aim. Acute myocardial infarction represents the vital cause of cardiac death, and many measurable biomarkers have been reported to be related to the prognosis of acute myocardial infarction. Our study was to investigate the role of a novel biomarker, the combination of platelet count, and neutrophil-to-lymphocyte ratio, for predicting in-hospital and long-term mortality of aged patients with acute myocardial infarction. Method. This was a study recording 637 patients who were diagnosed with acute myocardial infarction. Our patients were grouped according to the combination of platelet count and neutrophil-to-lymphocyte ratio. The prognostic role of the combination of platelet count and neutrophil-to-lymphocyte ratio on mortality was assessed by the univariate and multivariate Cox regression analysis. Result. Our study population was divided into three parts according to the median values of platelet count and neutrophil-to-lymphocyte ratio. It was indicated that platelet count and neutrophil-to-lymphocyte ratio were correlative mutually to a certain degree $(p=0.010)$. The Kaplan-Meier analysis showed that the combination of high platelet count and high neutrophil-tolymphocyte ratio had a greater risk of death in short- and long-term endpoints (log-rank $p=0.046, p<0.001$, respectively). Moreover, by multivariate analysis, both high platelet count and high neutrophil-to-lymphocyte ratio groups were an independent predictor (hazard ratio: $2.132,95 \%$ confidence interval: $1.020-4.454, p=0.044$ ) and long-term mortality (hazard ratio: $2.791,95 \%$ confidence interval: $1.406-5.538, p=0.003$ ). Conclusion. The combination of platelet count and neutrophil-to-lymphocyte ratio could be a useful predictor for the prediction of in-hospital and long-term mortality in aged patients with acute myocardial infarction.
\end{abstract}

\section{Introduction}

Acute myocardial infarction (AMI) is the leading cause of cardiac death, especially for the aged people $[1,2]$. Along with the prolongation of the life expectancy in the elderly population, cardiac death is more frequent in patients aged over 65 years old $[3,4]$. The severe condition with insidious onset is one of the reasons for the increasing mortality in elderly AMI patients $[3,5,6]$. The current dilemma for clinical workers is figuring out a simple and powerful prognostic biomarker to identify high-risk patients.

Clinical and experimental evidence found that inflammation played a crucial role in the development and progression of AMI [7-9]. Previous studies showed that inflammatory markers, such as platelet count (PLC) and neutrophil-to-lymphocyte ratio (NLR), were connected with 
poor clinical outcomes in patients with AMI $[10,11]$. Pathologically increased PLC in peripheral blood contributed to the initiation, progression, and complication of atherosclerosis [12-14]. According to a population-based cohort study, elevated PLC was associated with higher mortality and increased risk of cardiovascular events in AMI patients [15]. However, previous conflicting results showed that there was no correlation between elevated PLC and poorer cardiovascular endpoints [16-18].

Neutrophils and lymphocytes mirror the inflammatory status and have an effect on the destabilization of atherosclerotic plaque [19]. Accordingly, NLR has been assessed as a prognostic biomarker for cardiovascular diseases. A retrospective study by Han et al. showed that elevated NLR was a useful marker to predict long-term all-cause death in patients with AMI [20]. Nonetheless, the predictive value of NLR varies slightly in different studies, which is an imperfection for the risk stratification in AMI patients [21, 22].

Thus, to optimize the predictive value of PLC and NLR, some studies evaluated an original inflammation-related prognostic marker, named the combination of PLC and NLR (COP-NLR) $[23,24]$. These studies showed that COP-NLR was a better prognostic predictor in patients with inflammatory diseases $[23,24]$. However, to the best of our knowledge, the usefulness of COP-NLR has not been evaluated in AMI. Therefore, in the present study, we intended to investigate the prognostic value of COP-NLR in aged AMI patients.

\section{Methods}

2.1. Participants. This was a retrospective study which reviewed the data of 637 patients with AMI at the Tongji Hospital, Tongji Medical College, Huazhong University of Science and Technology, between January 2015 and October 2017. AMI was defined as either ST-segment elevation myocardial infarction (STEMI, typical symptoms of myocardial ischemia lasting for $>30 \mathrm{~min}$, with ST-segment elevation $>1 \mathrm{~mm}$ in $\geq 2$ contiguous standard or precordial leads and/or new onset of left bundle branch block) [25] and without ST-segment elevation myocardial infarction (NSTEMI, a rise of myocardial injury markers in combination with typical angina pectoris and without ST-segment elevation) [26]. The exclusion criteria were as follows: (a) all patients who were younger than 65 or older than 85 years; (b) patients with sepsis or trauma; (c) patients who were diagnosed with active cancer, autoimmune diseases, hematological proliferative diseases, chronic pulmonary disease, renal failure, and end-stage liver disease; and (d) patients who received steroid therapy or chemotherapy around the diagnosis index during six months.

This study has got approval by the ethics committee of Tongji Hospital, Tongji Medical College, Huazhong University of Science and Technology (TJ-C20141112), and is in accordance with the Helsinki Declaration.

2.2. Study Procedures and Laboratory Analysis. Venous blood samples were collected from all patients at the time of admission. Collection patients' demographic data were documented during hospitalization, including vital signs at admission, risk factors, medical history, and drug use. Laboratory results included creatinine, aminopherase, $\mathrm{N}$-terminal prohormone of brain natriuretic peptide (NTProBNP), cardiac troponin I (CTnI), total cholesterol (TC), triglycerides, high-density lipoprotein cholesterol (HDL), and low-density lipoprotein cholesterol (LDL). The NLR was calculated as the ratio of the neutrophils to lymphocytes. Besides, echocardiographic parameters included left ventricular ejection fraction (LVEF).

Angiographic data including thrombolysis in myocardial infarction trial blood flow grade (TIMI) and Gensini score during the in-hospital period were obtained from the electronic medical records database. All included patients underwent coronary angiography. And the results of coronary angiography were determined by two professional cardiovascular doctors. Based on the results of coronary angiography and clinical findings, doctors selected using different treatment strategies according to the guideline [27]. In the perioperative period, anticoagulants, heparin/low molecular heparin, and tirofiban were used. For the patients with coronary stent placement, they were treated with aspirin and clopidogrel (or ticagrelor) as adjuvant antiplatelet therapy.

2.3. Study Endpoint and Follow-Up. The endpoints of our study were all-cause mortality that happened in-hospital and during the follow-up period. All-cause death was defined as mortality from any cause. The data during the hospitalization were obtained from the hospital administration system. And the information during follow-up was regularly collected from hospital records or the telephone interviews with patients or their relatives.

2.4. Statistical Analysis. In baseline characteristics analysis, continuous variables were presented as mean \pm standard deviation and tested for normal distribution by the Kolmogorov-Smirnov test. The sample size of 224 patients was calculated to give a power of $90 \%$, a type I error of 0.05 , and a $10 \%$ drop-out rate. Comparative analyses between groups for continuous variables were performed by Student's $t$-test or Mann-Whitney $U$ test. Categorical variables were summarized as percentages and compared with the $\chi^{2}$ test. Correlation between PLC and NLR was tested using the Spearman correlation coefficient. The mortality according to the median values of PLC and NLR were analyzed by the Kaplan-Meier method, and results were compared using the log-rank test. Univariate and multivariate analyses were performed to determine the significance of prognostic variables using the Cox proportional hazards model. Any variables examined in the univariate analysis for which the $p$ value was $<0.10$ were contained in the multivariate model. A value of $p<0.05$ was supposed to be statistically significant.

\section{Results}

3.1. Baseline Characteristics. A total of 637 patients with AMI were enrolled in this study. A detailed description of demographic and laboratory characteristics is presented in 
Table 1. Median values of PLC and NLR were 198.00 and 4.88 , respectively. There was a weak but negative correlation between PLC and NLR (Spearman $r=-0.102, p=0.010$; Figure 1). The patients were divided into 3 groups: low PLC $(\mathrm{PLC}<198.00)$ and low NLR $(\mathrm{NLR}<4.88)(n=145)$ versus either low PLC or low NLR $(n=351)$ versus high PLC $(\mathrm{PLC} \geq 198.00)$ and high NLR $(\mathrm{NLR} \geq 4.88)(n=141)$, as noted in Table 1. Both groups were predominantly male. The mean age of patients was $72.40 \pm 5.28,71.95 \pm 5.20$, and $72.31 \pm 4.83$ years, respectively, with an age range from 65 to 85 years. The high PLC and high NLR group had shorter reperfusion time $(p<0.001)$, longer hospitalization day, $(p=0.024)$, and worse heart function $(p<0.001)$.

As shown in Table 2, in terms of the angiographic and procedural characteristics, no significant differences were discovered in the Gp IIbIIIa inhibitor use, stent use, TIMI grade, and Gensini score between the three groups, except for the culprit vessel quantity and the use of thrombus aspiration.

3.2. Clinical Outcomes. During hospitalization and the average follow-up period was $771.71 \pm 16.39$ days, the all-cause mortality was significantly higher in patients with both high PLC and high NLR group ( $p=0.013$ for in-hospital mortality, $p<0.001$ for long-term mortality; Figure 2). The Kaplan-Meier curves based on the median values of NLR and PLC are shown in Figure 3. When PLC and NLR were compared between patients with and without death during short- and long-term follow-up, NLR was significantly higher in patients with death (both log-rank: $p<0.001$; Figures 3(a) and 3(b)). However, there was no significant difference in PLC (log-rank: $p=0.478$ and $p=0.482$, resp.; Figures 3(c) and 3(d)). The Kaplan-Meier survival analysis according to the combination of PLC and NLR is shown in Figure 4 . The mortality increased significantly in the high PLC and high NLR group during hospitalization (log-rank: $p=0.046$; Figure 4(a)) and long-term follow-up (log-rank: $p<0.001$, Figure 4(b)).

3.3. Receiver Operating Characteristic Curve (ROC) Analysis. As shown in Figure 5, the distinction does not prove a statistical significance in ROC analysis of PLC ( $p=0.361$, Figure 5(a)). The area under the curve of the NLR with the outcomes of mortality was 0.677 (95\% confidence interval [CI]: $0.626-0.728, p<0.001$; sensitivity $=75.00 \%$, specificity $=56.40 \%$; Figure $5(\mathrm{~b}))$.

3.4. Independent Predictors of Mortality. In multivariate regression analysis, Killip class (hazard ratio [HR]: 1.810, 95\% CI: 1.367-2.397, $p<0.001$ ), LVEF (HR: 0.978, 95\% CI: $0.957-0.999, p=0.040)$, history of coronary heart disease (CHD) (HR: 2.195, 95\% CI: 1.130-4.264, $p=0.020$ ), neutrophil count (HR: 1.115, 95\% CI: 1.044-1.190, $p=0.001$ ), HDL (HR: $0.311,95 \%$ CI: $0.117-0.824, p=0.019$ ), and the combination of high PLC and high NLR (HR: 2.132, 95\% CI: $1.020-4.454, p=0.044$ ) were found as independent predictors of in-hospital mortality (Table 3).
The combination of high PLC and high NLR was also found as an independent predictor of long-term mortality (HR: 2.791, 95\% CI: $1.406-5.538, p=0.003$ ) along with age (HR: $1.066,95 \%$ CI: $1.020-1.115, p=0.005$ ), Killip class (HR: 1.490 , 95\% CI: $1.165-1.905, p=0.001$ ), LVEF (HR: 0.974, 95\% CI: 0.956-0.992, $p=0.005$ ), and creatinine (HR: 1.006, 95\% CI: $1.000-1.011, p=0.035$ ) (Table 4).

\section{Discussion}

The inflammatory process has a central role in the development of atherosclerosis, instability of atherosclerotic plaques, and formation of thrombus [28-30]. The role of inflammatory markers on the prognosis of AMI has been suggested in previous studies [31, 32]. A large study of postmenopausal women showed that high PLC was associated with increased cardiovascular mortality [33]. Previous studies revealed that an elevated level of NLR was related to increased cardiovascular risk in patients with AMI $[34,35]$. However, in the current study, PLC had no predictive significance in univariate or multivariate analysis. Some case-control studies and observational studies have also shown that there was no association between high PLC and risk of death [18, 36]. In the present study, NLR had a strong association with death in a patient with AMI. Yet, our results showed that NLR was not an independent predictor of short- and long-term mortality. Thus, further clinical studies are needed to explore a better biomarker to predict the death prognosis in AMI patients.

Recent studies found that the interaction of platelet and leukocyte is involved in the complex pathologic process of atherosclerosis [37]. Platelet-leukocyte interactions also activated cytokine expression, caused adhesion of endothelial cell, and promoted arterial thrombosis [38]. Based on this interaction of platelet with leukocyte, we made further research for the prognostic value of the combination of PLC and NLR in aged patients with AMI. The COP-NLR consisted of two biomarkers (PLC and NLR) related to cellular inflammation. It may reflect the balance of inflammation in the body more comprehensively, has stronger predictive potential for clinical outcomes, and contributes more to the risk stratification. COP-NLR has been identified as a novel predictive factor in patients with inflammatory diseases in previous studies $[23,24]$. Our study showed that an increased level of COP-NLR was associated with a higher risk of in-hospital and long-term mortality in aged patients with AMI. Compared with individual PLC or NLR, the COP-NLR was a stronger predictor of mortality in aged AMI patients.

Although the pathologic mechanism between thrombocytosis and death prognosis has not been fully understood, several potential mechanisms have been described. Increased release of inflammatory mediators might lead to higher PLC [39]. The elevated platelet concentration represented greater possibilities to attach to the vessel wall, leading to platelet-dependent thrombus formation. In addition, on vulnerable coronary plaques, forming platelet-rich thrombi resulted in worse outcomes [40, 41].

The high NLR might probably be due to an increased neutrophil count and a decreased lymphocyte count. After the onset of AMI, increased cytokines, such as TNF- $\alpha$ and IL-6 
TABLE 1: Baseline characteristics.

\begin{tabular}{|c|c|c|c|c|}
\hline Characteristics & $\begin{array}{l}\text { Low PLC and low NLR } \\
\qquad(n=145)\end{array}$ & $\begin{array}{l}\text { Either low PLC or low NLR } \\
\qquad(n=351)\end{array}$ & $\begin{array}{l}\text { High PLC and high NLR } \\
\qquad(n=141)\end{array}$ & $p$ \\
\hline Age, year & $72.40 \pm 5.28$ & $71.95 \pm 5.20$ & $72.31 \pm 4.83$ & 0.615 \\
\hline Gender (male), $n$ (\%) & $105(72.41)$ & $227(64.67)$ & $85(60.28)$ & 0.088 \\
\hline Smoke and drink, $n(\%)$ & $59(40.69)$ & $140(39.89)$ & $54(38.30)$ & 0.914 \\
\hline Hypertension, $n(\%)$ & $85(58.62)$ & $205(58.40)$ & $89(63.12)$ & 0.610 \\
\hline Prior CHD, $n(\%)$ & $23(15.86)$ & $37(10.54)$ & $12(8.51)$ & 0.116 \\
\hline Diabetes, $n(\%)$ & $35(24.14)$ & $93(26.50)$ & $38(26.95)$ & 0.831 \\
\hline Stroke, $n(\%)$ & $15(10.34)$ & $54(15.38)$ & $16(11.35)$ & 0.237 \\
\hline $\mathrm{SBP}$ on admission $(\mathrm{mmHg})$ & $130.99 \pm 24.30$ & $130.01 \pm 24.07$ & $125.53 \pm 25.94$ & 0.117 \\
\hline $\begin{array}{l}\text { DBP on admission } \\
(\mathrm{mmHg})\end{array}$ & $75.18 \pm 14.22$ & $75.61 \pm 14.09$ & $74.77 \pm 16.14$ & 0.806 \\
\hline $\begin{array}{l}\text { HR on admission (beats/ } \\
\text { min) }\end{array}$ & $73.43 \pm 11.25$ & $77.08 \pm 15.59$ & $82.74 \pm 19.67$ & $<0.001$ \\
\hline Pain to reperfusion $(\mathrm{h})$ & $16.70 \pm 8.14$ & $14.16 \pm 8.99$ & $12.32 \pm 8.27$ & $<0.001$ \\
\hline Hospitalization day & $8.28 \pm 5.12$ & $8.44 \pm 6.43$ & $10.21 \pm 9.50$ & 0.024 \\
\hline Killip class, III-IV, $n(\%)$ & $11(7.59)$ & $50(14.25)$ & $37(26.24)$ & $<0.001$ \\
\hline \multicolumn{5}{|l|}{ Diagnosis } \\
\hline STEMI & $57(39.31)$ & $209(59.54)$ & $95(67.38)$ & $<0.001$ \\
\hline NSTEMI & $88(60.69)$ & $142(40.46)$ & $46(32.62)$ & $<0.001$ \\
\hline LVEF (\%) & $55.86 \pm 12.33$ & $52.72 \pm 12.21$ & $49.92 \pm 12.27$ & $<0.001$ \\
\hline Creatinine $(\mu \mathrm{mol} / \mathrm{l})$ & $87.10 \pm 26.91$ & $92.98 \pm 39.34$ & $93.60 \pm 35.44$ & 0.202 \\
\hline $\operatorname{AST}(\mathrm{u} / \mathrm{l})$ & $55.45 \pm 68.72$ & $90.26 \pm 106.13$ & $136.06 \pm 129.10$ & $<0.001$ \\
\hline $\operatorname{ALT}(\mathrm{u} / \mathrm{l})$ & $28.68 \pm 39.30$ & $36.15 \pm 37.78$ & $43.52 \pm 44.94$ & 0.007 \\
\hline NT-proBNP (pg/ml) & $3863.64 \pm 6575.65$ & $4813.60 \pm 7299.85$ & $5702.91 \pm 11031.69$ & 0.161 \\
\hline $\mathrm{CTnI}(\mathrm{pg} / \mathrm{ml})$ & $10058.40 \pm 15443.85$ & $14747.61 \pm 18692.72$ & $22783.39 \pm 20504.55$ & $<0.001$ \\
\hline $\mathrm{HDL}(\mathrm{mmol} / \mathrm{l})$ & $1.09 \pm 0.41$ & $1.08 \pm 0.44$ & $1.17 \pm 0.50$ & 0.124 \\
\hline $\mathrm{LDL}(\mathrm{mmol} / \mathrm{l})$ & $2.52 \pm 0.92$ & $2.52 \pm 0.92$ & $2.79 \pm 1.09$ & 0.012 \\
\hline Total triglyceride $(\mathrm{mmol} / \mathrm{l})$ & $1.36 \pm 0.81$ & $1.39 \pm 1.09$ & $1.20 \pm 1.08$ & 0.169 \\
\hline Total cholesterol $(\mathrm{mmol} / \mathrm{l})$ & $4.00 \pm 1.07$ & $4.04 \pm 1.09$ & $4.42 \pm 1.25$ & 0.001 \\
\hline WBC count $\left(10^{9} / \mathrm{l}\right)$ & $6.97 \pm 2.35$ & $9.00 \pm 3.19$ & $12.37 \pm 4.58$ & $<0.001$ \\
\hline $\operatorname{PLC}\left(10^{9} / 1\right)$ & $153.80 \pm 26.76$ & $201.10 \pm 65.48$ & $258.08 \pm 62.10$ & $<0.001$ \\
\hline Neutrophil count $\left(10^{9} / 1\right)$ & $4.62 \pm 1.86$ & $6.97 \pm 3.32$ & $10.70 \pm 4.29$ & $<0.001$ \\
\hline Lymphocyte count $\left(10^{9} / 1\right)$ & $1.67 \pm 0.63$ & $1.42 \pm 0.68$ & $1.02 \pm 0.47$ & $<0.001$ \\
\hline Monocyte count $\left(10^{9} / 1\right)$ & $0.52 \pm 0.20$ & $0.56 \pm 0.28$ & $0.61 \pm 0.39$ & 0.025 \\
\hline Hemoglobin $(\mathrm{mg} / \mathrm{dl})$ & $129.19 \pm 18.56$ & $126.94 \pm 19.25$ & $123.43 \pm 17.86$ & 0.033 \\
\hline NLR & $2.92 \pm 1.03$ & $6.61 \pm 6.35$ & $12.29 \pm 7.17$ & $<0.001$ \\
\hline \multicolumn{5}{|c|}{ Medications in hospital, $n(\%)$} \\
\hline Aspirin & $140(96.55)$ & $338(96.30)$ & $132(93.62)$ & 0.356 \\
\hline Clopidogrel & $135(93.10)$ & $337(96.01)$ & $131(92.91)$ & 0.244 \\
\hline Beta-blocker & $108(74.48)$ & $250(71.23)$ & $94(66.67)$ & 0.342 \\
\hline ACEI/ARB & $109(75.17)$ & $244(69.52)$ & $92(65.25)$ & 0.184 \\
\hline Statin & $144(99.31)$ & $343(97.72)$ & $136(96.45)$ & 0.254 \\
\hline
\end{tabular}

Mean \pm SD and $n$ (\%) are reported for continuous and categorical variables, respectively. Abbreviations: SD, standard deviation; PLC, platelet count; NLR, neutrophil-to-lymphocyte ratio; COP-NLR, combination of platelet count and neutrophil-to-lymphocyte ratio; CHD, coronary heart disease; SBP, systolic blood pressure; DBP, diastolic blood pressure; HR, heart rate; STEMI, ST-segment elevation myocardial infarction; NSTEMI, non-ST-elevation myocardial infarction; LVEF, left ventricular ejection fraction; AST, aspartate aminotransferase; ALT, alanine aminotransferase; NT-proBNP, N-terminal probrain natriuretic peptide; CTnI, cardiac troponin I; HDL, high-density lipoprotein cholesterol; LDL, low-density lipoprotein cholesterol; WBC, white blood cell; SII, systemic immune-inflammatory index; ACEI/ARB, angiotensin-converting enzyme inhibitor/angiotensin II receptor blocker.

caused relative neutrophilia [42]. Increased neutrophil counts predicted larger infarct size and adverse long-term cardiac prognosis [43]. Conversely, it was suggested that lymphocytopenia had a connection with AMI [44]. Similarly, Shiyovich and colleagues' study showed that there was a significant negative linear association between lymphocytes and death in the long-term follow-up period [45]. Lower lymphocyte counts might be owing to an abruptly increased level of corticosteroids and increased inflammation-related lymphocytes apoptosis $[46,47]$. The NLR integrated for two cellular subtypes with opposite actions when it comes to vascular inflammation. According to Ayca's study, preprocedural NLR > 4.9 was significantly related to stent thrombosis and higher mortality in patients with AMI [22]. However, the predictive value of NLR varied slightly in different studies [21, 22]. This was probably because of the differences in study design, population selection, statistical methods, outcome measurement.

It was indicated that LVEF and Killip class were important parameters for predicting adverse outcomes [48, 49]. Our study showed a similar result for predicting in-hospital 


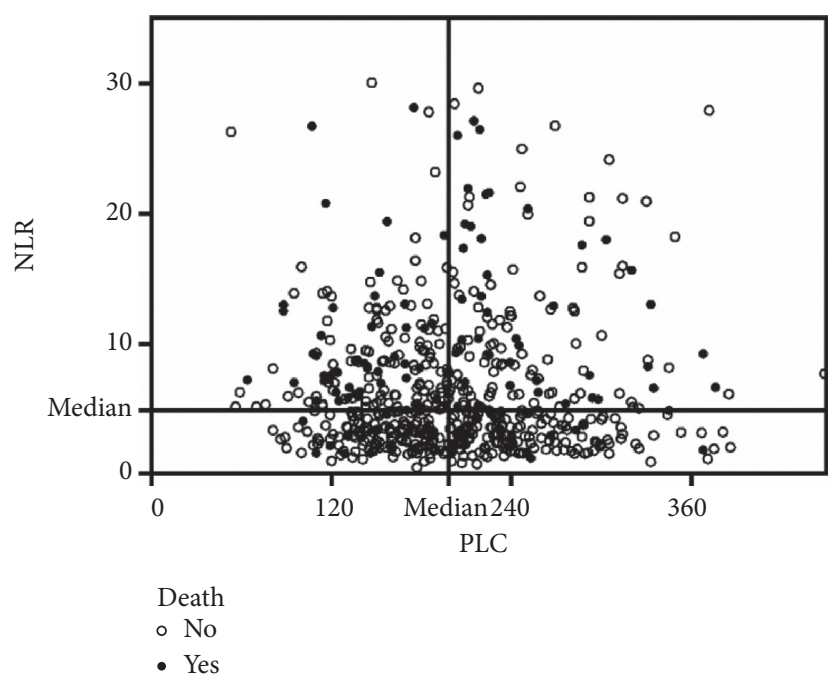

Figure 1: Correlation between PLC and NLR. When PLC cutoff value of 198.00 and NLR cutoff value of 4.88 were used, $34.04 \%$ mortality occurred in the high PLC and high NLR group (right upper quadrant). There was a weak but negative correlation between PLC and NLR: $r=-0.102, p=0.010$. Abbreviations: PLC, platelet count; NLR, neutrophil-to-lymphocyte ratio.

TABLE 2: Angiographic and procedural characteristics in the study population.

\begin{tabular}{|c|c|c|c|c|}
\hline Characteristics & $\begin{array}{l}\text { Low PLC and low NLR } \\
\qquad(n=145)\end{array}$ & $\begin{array}{c}\text { Either low PLC or low NLR } \\
(n=351)\end{array}$ & $\begin{array}{l}\text { High PLC and high NLR } \\
\qquad(n=141)\end{array}$ & $p$ \\
\hline Culprit vessel, $n$ (\%) & & & & 0.102 \\
\hline LAD & $130(89.66)$ & $305(86.89)$ & $123(87.23)$ & \\
\hline LCX & $98(67.59)$ & $225(64.10)$ & $85(60.28)$ & \\
\hline RCA & $103(71.03)$ & $216(61.54)$ & $92(65.25)$ & \\
\hline Number of diseased vessels, $n(\%)$ & & & & $<0.001$ \\
\hline 0 & $1(0.69)$ & $0(0.00)$ & $0(0.00)$ & \\
\hline 1 & $27(18.62)$ & $101(28.77)$ & $43(30.50)$ & \\
\hline 2 & $47(32.41)$ & $105(29.91)$ & $37(26.24)$ & \\
\hline 3 & $70(48.28)$ & $145(41.31)$ & $61(43.26)$ & \\
\hline $\begin{array}{l}\text { Use of thrombus aspiration, } n \\
\text { (\%) }\end{array}$ & $7(4.83)$ & $34(9.69)$ & $27(19.15)$ & $<0.001$ \\
\hline Gp IIbIIIa inhibitor use, $n(\%)$ & $124(85.52)$ & $293(83.48)$ & $112(79.43)$ & 0.371 \\
\hline Stent use, $n(\%)$ & $114(78.62)$ & $264(75.21)$ & $98(69.50)$ & 0.197 \\
\hline Preprocedural TIMI grade, $n$ (\%) & & & & 1.000 \\
\hline 0 & $73(50.34)$ & $223(63.53)$ & $98(69.50)$ & \\
\hline 1 & $65(44.83)$ & $116(33.05)$ & $38(26.95)$ & \\
\hline 2 & $6(4.14)$ & $10(2.85)$ & $5(3.55)$ & \\
\hline 3 & $1(0.69)$ & $2(0.57)$ & $0(0.00)$ & \\
\hline Postprocedural TIMI grade, $n$ (\%) & & & & 1.000 \\
\hline 0 & $10(6.90)$ & $50(14.25)$ & $12(8.51)$ & \\
\hline 1 & $9(6.21)$ & $18(5.13)$ & $21(14.89)$ & \\
\hline 2 & $5(3.45)$ & $16(4.56)$ & $4(2.84)$ & \\
\hline 3 & $121(83.45)$ & $267(76.07)$ & $104(73.76)$ & \\
\hline Gensini score (SD) & $98.86(60.95)$ & $89.62(46.64)$ & $98.26(57.20)$ & 0.103 \\
\hline
\end{tabular}

Mean (SD) and $n$ (\%) are reported for continuous and categorical variables, respectively. Abbreviations: SD, standard deviation; PLC, platelet count; NLR, neutrophil-to-lymphocyte ratio; COP-NLR, combination of platelet count and neutrophil-to-lymphocyte ratio; LAD, left coronary artery; LCX, left circumflex; RCA, right coronary artery; TIMI, thrombolysis in myocardial infarction.

and long-term prognosis of AMI. Along with COP-NLR, we also identified LVEF and Killip class as independent predictors of clinical outcomes in patients with AMI. Thus, this observation may support that the prognosis for patients with AMI is likely to be multifactorial. The identification of the potential predictor may provide a certain clinical assist for the risk stratification of AMI patients.
In the present study, our results showed that the COPNLR was an independent predictor of all-cause mortality in AMI. This is the first report to investigate the value of combined PLC and NLR to predict the in-hospital and longterm mortality in aged AMI patients. Both PLC and NLR could be easily and widely available, calculated from WBC subtype counts, which were routinely performed at 


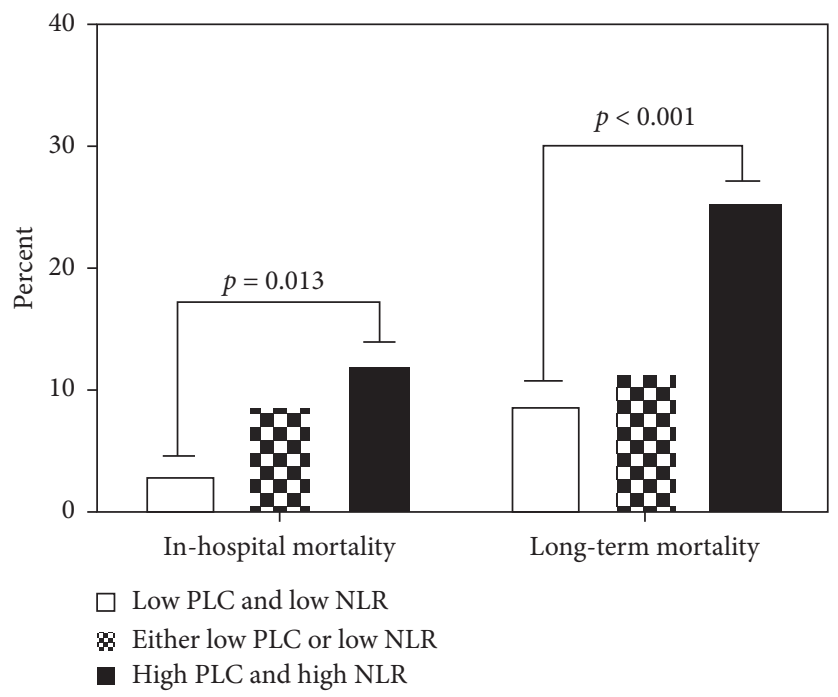

Figure 2: Clinical outcomes in 3 groups between in-hospital and long-term follow-up. Patients in the high PLC and high NLR group had significantly higher rates of all-cause mortality. Abbreviations: PLC, platelet count; NLR, neutrophil-to-lymphocyte ratio.

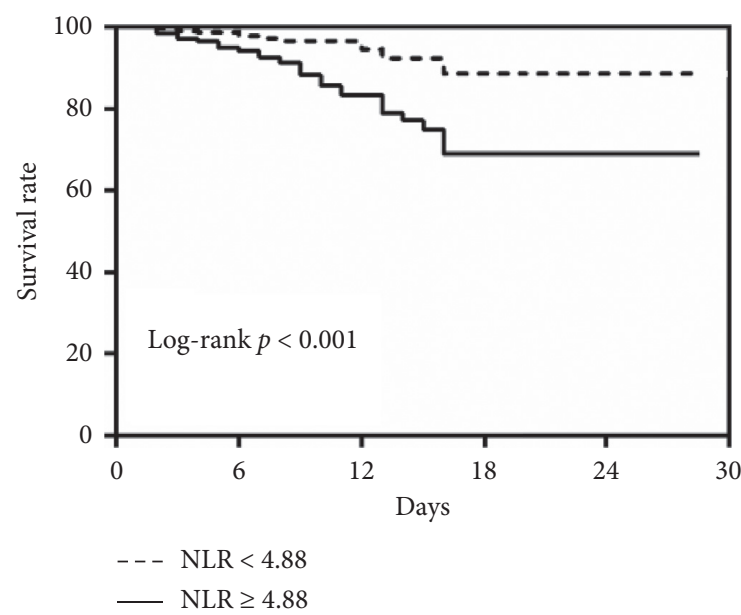

(a)

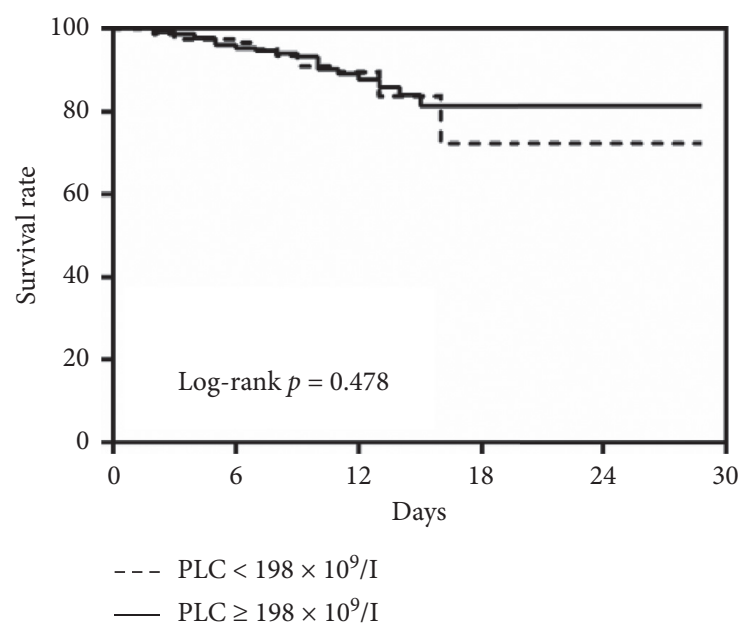

(c)

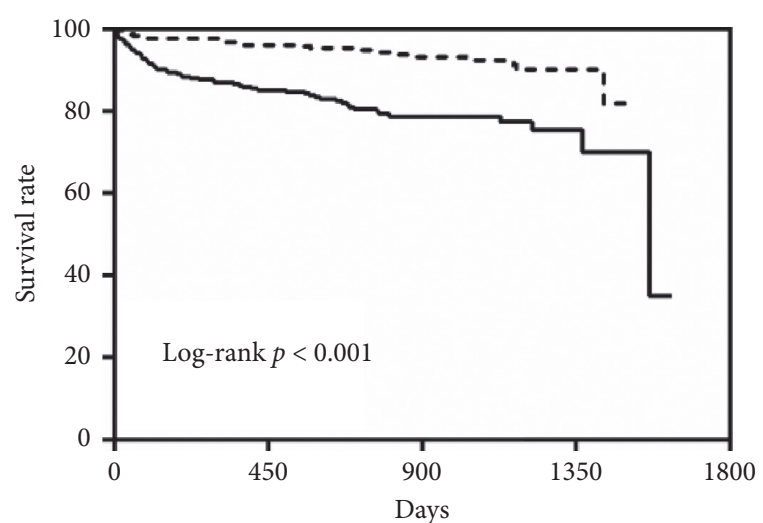

$---\operatorname{NLR}<4.88$

- NLR $\geq 4.88$

(b)

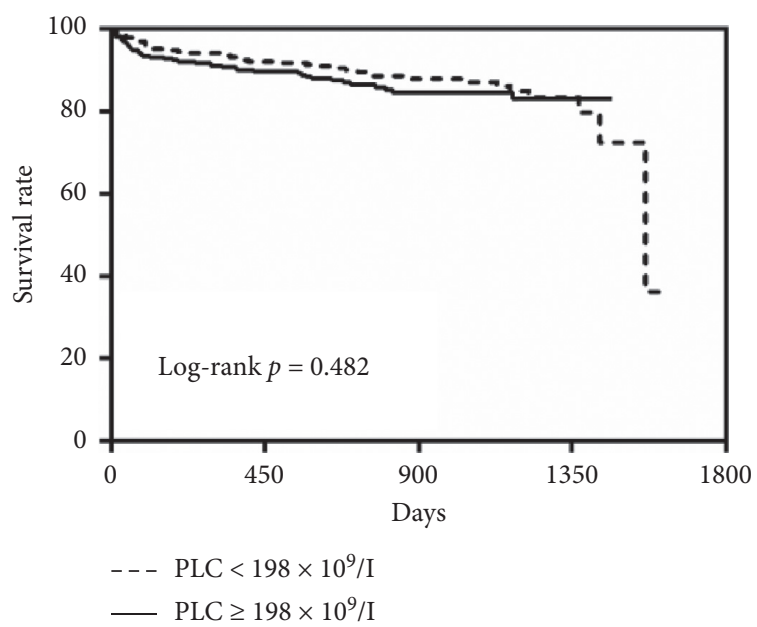

(d)

Figure 3: Kaplan-Meier survival curve of all-cause mortality based on the median value of NLR and PLC. (a) In-hospital mortality stratified by NLR. (b) Long-term mortality stratified by NLR. (c) In-hospital mortality stratified by PLC. (d) Long-term mortality stratified by PLC. Abbreviations: PLC, platelet count; NLR, neutrophil-to-lymphocyte ratio. 


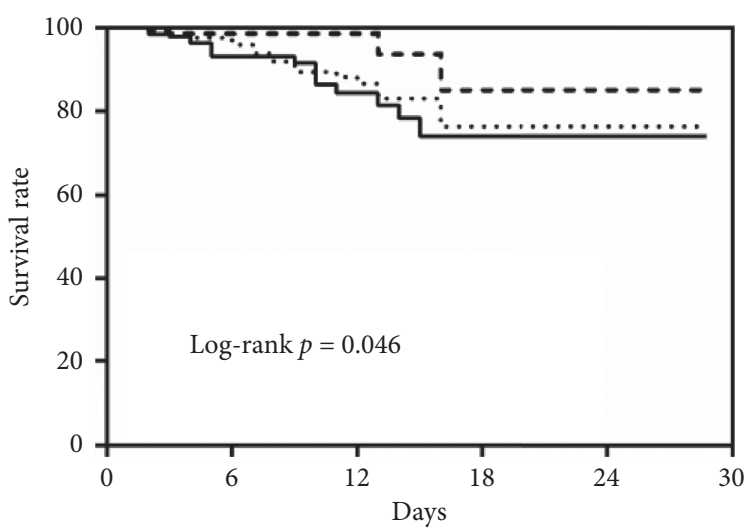

- - - Low PLC and low NLR

Either low PLC or low NLR

- High PLC and high NLR

(a)

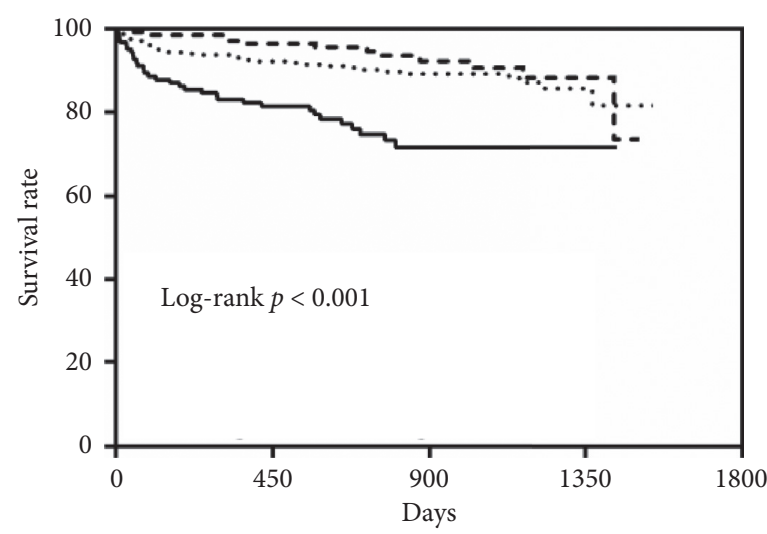

- - - Low PLC and low NLR

Either low PLC or low NLR

— High PLC and high NLR

(b)

FIgURE 4: The all-cause mortality based on the combination of PLC and NLR. (a) Kaplan-Meier survival curve of in-hospital mortality. (b) Kaplan-Meier survival curve of long-term mortality. Abbreviations: PLC, platelet count; NLR, neutrophil-to-lymphocyte ratio.

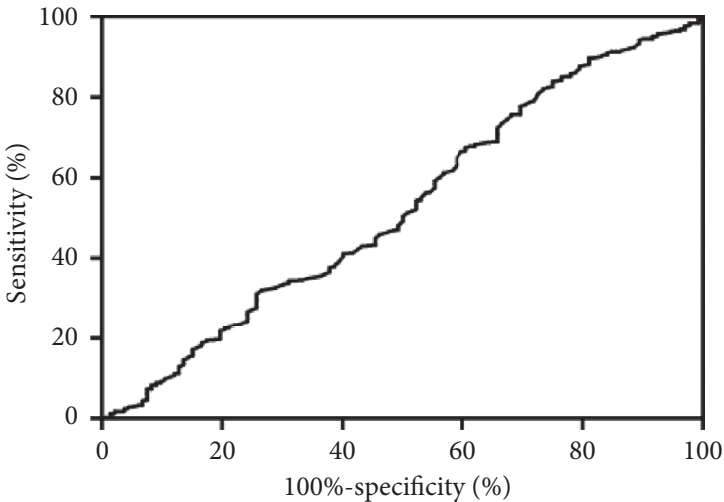

(a)

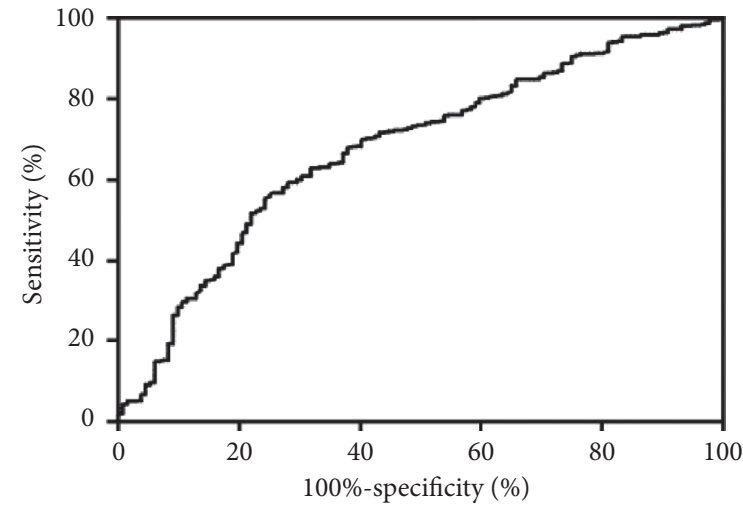

(b)

FIgURE 5: The ROC analysis of PLC and NLR. (a) ROC curves of PLC for mortality ( $p=0.361$ ). (b) ROC curves of NLR for mortality (area under the curve $=0.677 ; 95 \%$ confidence interval: $0.626-0.728, p<0.001)$. Abbreviations: ROC, receiver operating characteristic curve; PLC, platelet count; NLR, neutrophil-to-lymphocyte ratio.

TABle 3: Predictors of in-hospital mortality in univariable and multivariable Cox regression analyses.

\begin{tabular}{|c|c|c|c|c|c|c|}
\hline \multirow{2}{*}{ Variables } & \multicolumn{3}{|c|}{ Univariable } & \multicolumn{3}{|c|}{ Multivariable } \\
\hline & $\mathrm{HR}$ & $95 \% \mathrm{CI}$ & $p$ & HR & $95 \% \mathrm{CI}$ & $p$ \\
\hline Age & 1.026 & $0.975-1.079$ & 0.331 & & & \\
\hline Gender (male) & 1.457 & $0.838-2.533$ & 0.182 & & & \\
\hline Gensini score & 1.007 & $1.002-1.011$ & 0.005 & NS & NS & NS \\
\hline Killip class & 2.282 & $1.779-2.927$ & $<0.001$ & 1.810 & $1.367-2.397$ & $<0.001$ \\
\hline LVEF & 0.954 & $0.935-0.974$ & $<0.001$ & 0.978 & $0.957-0.999$ & 0.040 \\
\hline Smoke and drink & 1.521 & $0.832-2.779$ & 0.173 & & & \\
\hline Hypertension & 1.075 & $0.615-1.878$ & 0.800 & & & \\
\hline Prior CHD & 2.274 & $1.192-4.338$ & 0.013 & 2.195 & $1.130-4.264$ & 0.020 \\
\hline Diabetes & 1.106 & $0.618-1.979$ & 0.734 & & & \\
\hline Total cholesterol & 0.834 & $0.649-1.070$ & 0.153 & & & \\
\hline Total triglyceride & 0.932 & $0.691-1.255$ & 0.641 & & & \\
\hline $\mathrm{HDL}$ & 0.318 & $0.115-0.877$ & 0.027 & 0.311 & $0.117-0.824$ & 0.019 \\
\hline LDL & 0.918 & $0.691-1.220$ & 0.554 & & & \\
\hline Creatinine & 1.010 & $1.005-1.016$ & $<0.001$ & NS & NS & NS \\
\hline
\end{tabular}


TABLE 3: Continued.

\begin{tabular}{|c|c|c|c|c|c|c|}
\hline \multirow{2}{*}{ Variables } & \multicolumn{3}{|c|}{ Univariable } & \multicolumn{3}{|c|}{ Multivariable } \\
\hline & HR & $95 \% \mathrm{CI}$ & $p$ & HR & $95 \% \mathrm{CI}$ & $p$ \\
\hline WBC count & 1.119 & $1.062-1.180$ & $<0.001$ & NS & NS & NS \\
\hline Neutrophil count & 1.144 & $1.087-1.205$ & $<0.001$ & 1.115 & $1.044-1.190$ & 0.001 \\
\hline Lymphocyte count & 0.839 & $0.545-1.293$ & 0.426 & & & \\
\hline PLC $>198.00$ & 1.220 & $0.702-2.119$ & 0.481 & & & \\
\hline $\mathrm{NLR}>4.88$ & 1.045 & $1.019-1.072$ & 0.001 & NS & NS & NS \\
\hline PLC $>198.00$ and NLR $>4.88$ & 3.476 & $1.164-10.383$ & 0.026 & 2.132 & $1.020-4.454$ & 0.044 \\
\hline
\end{tabular}

HR, hazard ratio; CI, confidence interval; NS, no statistical significance; CHD, coronary heart disease; LVEF, left ventricular ejection fraction; HDL, highdensity lipoprotein cholesterol; LDL, low-density lipoprotein cholesterol; WBC, white blood cell; PLC, platelet count; NLR, neutrophil-to-lymphocyte ratio.

TABle 4: Predictors of long-term mortality in univariable and multivariable cox regression analyses.

\begin{tabular}{|c|c|c|c|c|c|c|}
\hline \multirow{2}{*}{ Variables } & \multicolumn{3}{|c|}{ Univariable } & \multicolumn{3}{|c|}{ Multivariable } \\
\hline & HR & $95 \% \mathrm{CI}$ & $p$ & HR & $95 \% \mathrm{CI}$ & $p$ \\
\hline Age & 1.082 & $1.039-1.126$ & $<0.001$ & 1.066 & $1.020-1.115$ & 0.005 \\
\hline Gender (male) & 1.487 & $0.954-2.317$ & 0.080 & NS & NS & NS \\
\hline Gensini score & 1.002 & $0.998-1.006$ & 0.225 & & & \\
\hline Killip class & 2.011 & $1.009-2.513$ & $<0.001$ & 1.490 & $1.165-1.905$ & 0.001 \\
\hline LVEF & 0.960 & $0.944-0.977$ & $<0.001$ & 0.974 & $0.956-0.992$ & 0.005 \\
\hline Smoke and drink & 1.110 & $0.705-1.747$ & 0.652 & & & \\
\hline Hypertension & 1.262 & $0.797-1.998$ & 0.321 & & & \\
\hline Prior CAD & 1.010 & $0.486-2.098$ & 0.978 & & & \\
\hline Diabetes & 1.372 & $0.848-2.218$ & 0.197 & & & \\
\hline Total cholesterol & 1.035 & $0.850-1.261$ & 0.729 & & & \\
\hline Total triglyceride & 0.992 & $0.784-1.255$ & 0.948 & & & \\
\hline HDL & 1.338 & $0.870-2.057$ & 0.185 & & & \\
\hline LDL & 1.037 & $0.825-1.303$ & 0.756 & & & \\
\hline Creatinine & 1.009 & $1.005-1.014$ & $<0.001$ & 1.006 & $1.000-1.011$ & 0.035 \\
\hline WBC count & 1.111 & $1.058-1.167$ & $<0.001$ & NS & NS & NS \\
\hline Neutrophil count & 1.118 & $1.066-1.173$ & $<0.001$ & NS & NS & NS \\
\hline Lymphocyte count & 0.564 & $0.376-0.847$ & 0.006 & NS & NS & NS \\
\hline PLC $>198.00$ & 1.001 & $0.998-0.004$ & 0.549 & & & \\
\hline $\mathrm{NLR}>4.88$ & 1.051 & $1.028-1.074$ & $<0.001$ & NS & NS & NS \\
\hline PLC $>198.00$ and NLR $>4.88$ & 3.695 & $1.893-7.212$ & $<0.001$ & 2.791 & $1.406-5.538$ & 0.003 \\
\hline
\end{tabular}

HR, hazard ratio; CI, confidence interval; NS, no statistical significance; CHD, coronary heart disease; LVEF, left ventricular ejection fraction; HDL, highdensity lipoprotein cholesterol; LDL, low-density lipoprotein cholesterol; WBC, white blood cell; PLC, platelet count; NLR, neutrophil-to-lymphocyte ratio.

admission. Because of its easy obtainment and low cost, COP-NLR is possible for clinical application.

We have to acknowledge that there are some limitations in this study. Firstly, the retrospective design of the study sets a limit to the convincement of our study. Due to the nature of our study, the results must be explained with caution, given the possibility of confounders. Secondly, our endpoints are so simple that we have not investigated the relationship between COP-NLR with other major adverse cardiovascular events, including reinfarction, target vessel revascularization, arrhythmia, cerebrovascular accident, and congestive heart failure. Thirdly, we could not compare COP-NLR with other conventional inflammatory markers, for instance, C-reactive protein, fibrinogen, or myeloperoxidase. Because they were not routinely obtained in our study population.

\section{Conclusion}

In conclusion, a higher level of COP-NLR was independently associated with poor prognosis in aged patients with AMI. The COP-NLR was a potential predictor for short- and long-term mortality in aged AMI patients.

\section{Data Availability}

The data used to support the findings of this study are available from the corresponding author upon request.

\section{Conflicts of Interest}

The authors declare that they have no conflicts of interest regarding this submission.

\section{Authors' Contributions}

Xiao-Qing Quan and Hong-Yan Ji contributed equally to this study.

\section{Acknowledgments}

This work was supported by the Shenzhen Key Medical Discipline Construction Fund (SZXK063) and National Natural Science Foundation of China (81400255). 


\section{References}

[1] E. J. Benjamin, S. S. Virani, C. W. Callaway et al., "Heart disease and stroke statistics-2018 update: a report from the American heart association," Circulation, vol. 137, no. 12, pp. e67-e492, 2018.

[2] S. Qiu, X. Cai, Z. Sun et al., "Heart rate recovery and risk of cardiovascular events and all-cause mortality: a meta-analysis of prospective cohort studies," Journal of the American Heart Association, vol. 6, no. 5, Article ID e005505, 2017.

[3] K. Kragholm, S. A. Goldstein, Q. Yang et al., "Trends in enrollment, clinical characteristics, treatment, and outcomes according to age in non-ST-segment-elevation acute coronary syndromes clinical trials," Circulation, vol. 133, no. 16, pp. 1560-1573, 2016.

[4] F. Wang, L.-Y. Zhang, P. Zhang et al., "Effect of physical activity on hospital service use and expenditures of patients with coronary heart disease: results from dongfeng-tongji cohort study in China," Current Medical Science, vol. 39, no. 3, pp. 483-492, 2019.

[5] H.-Y. Chen, D. D. McManus, J. S. Saczynski et al., "Characteristics, treatment practices, and in-hospital outcomes of older adults hospitalized with acute myocardial infarction," Journal of the American Geriatrics Society, vol. 62, no. 8, pp. 1451-1459, 2014.

[6] B. Azab, M. Zaher, K. F. Weiserbs et al., "Usefulness of neutrophil to lymphocyte ratio in predicting short- and longterm mortality after non-ST-elevation myocardial infarction," The American Journal of Cardiology, vol. 106, no. 4, pp. $470-476,2010$.

[7] D. W. Kehl, N. Iqbal, A. Fard, B. A. Kipper, A. De La Parra Landa, and A. S. Maisel, "Biomarkers in acute myocardial injury," Translational Research, vol. 159, no. 4, pp. 252-264, 2012.

[8] I. Mozos, C. Malainer, J. Horbanczuk et al., "Inflammatory markers for arterial stiffness in cardiovascular diseases," Frontiers in Immunology, vol. 8, p. 1058, 2017.

[9] R.-X. Xu, Y. Zhang, Y. Zhang et al., "Effects of pitavastatin on lipoprotein subfractions and oxidized low-density lipoprotein in patients with atherosclerosis," Current Medical Science, vol. 40, no. 5, pp. 879-884, 2020.

[10] G. Huang, X.-N. Zhong, B. Zhong et al., "Significance of white blood cell count and its subtypes in patients with acute coronary syndrome," European Journal of Clinical Investigation, vol. 39, no. 5, pp. 348-358, 2009.

[11] H. Mangge and G. Almer, "Immune-mediated inflammation in vulnerable atherosclerotic plaques," Molecules, vol. 24, no. 17, p. 3072, 2019.

[12] M. G. Alexandrakis, F. H. Passam, I. A. Moschandrea et al., "Levels of serum cytokines and acute phase proteins in patients with essential and cancer-related thrombocytosis," American Journal of Clinical Oncology, vol. 26, no. 2, pp. 135-140, 2003.

[13] K. Y. Stokes and D. N. Granger, "Platelets: a critical link between inflammation and microvascular dysfunction," The Journal of Physiology, vol. 590, no. 5, pp. 1023-1034, 2012.

[14] S. Karaman and A. Coskun, "Do MCHC, MPV, and procalcitonin levels determine prognosis in acute coronary syndrome?" Emergency Medicine International, vol. 2019, Article ID 6721279, 8 pages, 2019.

[15] G. Patti, G. Di Martino, F. Ricci et al., "Platelet indices and risk of death and cardiovascular events: results from a large population-based cohort study," Thrombosis and Haemostasis, vol. 119, no. 11, pp. 1773-1784, 2019.
[16] A. R. Folsom, W. D. Rosamond, E. Shahar et al., "Prospective study of markers of hemostatic function with risk of ischemic stroke," Circulation, vol. 100, no. 7, pp. 736-742, 1999.

[17] A. R. Folsom, K. K. Wu, W. D. Rosamond, A. R. Sharrett, and L. E. Chambless, "Prospective study of hemostatic factors and incidence of coronary heart disease," Circulation, vol. 96, no. 4, pp. 1102-1108, 1997.

[18] J. Klovaite, M. Benn, S. Yazdanyar, and B. G. Nordestgaard, "High platelet volume and increased risk of myocardial infarction: 39531 participants from the general population," Journal of Thrombosis and Haemostasis, vol. 9, no. 1, pp. 49-56, 2011.

[19] M. A. R. Muhmmed Suliman, A. A. Bahnacy Juma, A. A. Ali Almadhani, A. V. Pathare, S. S. A. Alkindi, and F. Uwe Werner, "Predictive value of neutrophil to lymphocyte ratio in outcomes of patients with acute coronary syndrome," $A r$ chives of Medical Research, vol. 41, no. 8, pp. 618-622, 2010.

[20] Y.-C. Han, T.-H. Yang, D.-I. Kim et al., "Neutrophil to lymphocyte ratio predicts long-term clinical outcomes in patients with ST-segment elevation myocardial infarction undergoing primary percutaneous coronary intervention," Korean Circulation Journal, vol. 43, no. 2, pp. 93-99, 2013.

[21] J. He, J. Li, Y. Wang, P. Hao, and Q. Hua, "Neutrophil-tolymphocyte ratio (NLR) predicts mortality and adverseoutcomes after ST-segment elevation myocardial infarction in Chinese people," International Journal of Experimental $\mathrm{Pa}$ thology, vol. 7, no. 7, pp. 4045-4056, 2014.

[22] B. Ayça, F. Akın, O. Celik et al., "Neutrophil to lymphocyte ratio is related to stent thrombosis and high mortality in patients with acute myocardial infarction," Angiology, vol. 66, no. 6, pp. 545-552, 2015.

[23] J. Wang, J. Qu, Z. Li et al., "Combination of platelet count and neutrophil-lymphocyte ratio as a prognostic marker to predict chemotherapeutic response and survival in metastatic advanced gastric cancer," Biomarkers in Medicine, vol. 11, no. 10, pp. 835-845, 2017.

[24] Y.-H. Lin, K.-P. Chang, Y.-S. Lin, and T.-S. Chang, "Pretreatment combination of platelet counts and neutrophil-lymphocyte ratio predicts survival of nasopharyngeal cancer patients receiving intensity-modulated radiotherapy," OncoTargets and Therapy, vol. 10, pp. 2751-2760, 2017.

[25] B. Ibanez, S. James, S. Agewall et al., "2017 ESC guidelines for the management of acute myocardial infarction in patients presenting with ST-segment elevation: the task force for the management of acute myocardial infarction in patients presenting with ST-segment elevation of the European society of cardiology (ESC)," European Heart Journal, vol. 39, no. 2, pp. 119-177, 2018.

[26] H. Jneid, D. Addison, D. L. Bhatt et al., "2017 AHA/ACC clinical performance and quality measures for adults with STelevation and non-ST-elevation myocardial infarction: a report of the American college of cardiology/American heart association task force on performance measures," Circ Cardiovasc Qual Outcomes, vol. 10, no. 10, Article ID e000032, 2017.

[27] P. Kolh, S. Windecker, F. Alfonso et al., "2014 ESC/EACTS guidelines on myocardial revascularization: the task force on myocardial revascularization of the European society of cardiology (ESC) and the European association for cardiothoracic surgery (EACTS). developed with the special contribution of the European association of percutaneous cardiovascular interventions (EAPCI)," European Heart Journal, vol. 35, no. 37, pp. 2541-2619, 2014. 
[28] K. H. Cho, M. H. Jeong, K. Ahmed et al., "Value of early risk stratification using hemoglobin level and neutrophil-tolymphocyte ratio in patients with ST-elevation myocardial infarction undergoing primary percutaneous coronary intervention," The American Journal of Cardiology, vol. 107, no. 6, pp. 849-856, 2011.

[29] S. Balta, T. Celik, D. P. Mikhailidis et al., "The relation between atherosclerosis and the neutrophil-lymphocyte ratio," Clinical and Applied Thrombosis/Hemostasis, vol. 22, no. 5, pp. 405-411, 2016.

[30] Z.-H. Ruan, Z.-X. Xu, X.-Y. Zhou, X. Zhang, and L. Shang, "Implications of necroptosis for cardiovascular diseases," Current Medical Science, vol. 39, no. 4, pp. 513-522, 2019.

[31] E. D. Coverstone, R. G. Bach, L. Chen et al., "A novel genetic marker of decreased inflammation and improved survival after acute myocardial infarction," Basic Research in Cardiology, vol. 113, no. 5, p. 38, 2018.

[32] A. R. Castro, S. O. Silva, and S. C. Soares, "The use of high sensitivity C-reactive protein in cardiovascular disease detection," Journal of Pharmacy \& Pharmaceutical Sciences, vol. 21, no. 1, pp. 496-503, 2018.

[33] G. C. Kabat, M. Y. Kim, A. K. Verma et al., "Platelet count and total and cause-specific mortality in the women's health initiative," Annals of Epidemiology, vol. 27, no. 4, pp. 274-280, 2017.

[34] S. R. Ommen, D. O. Hodge, R. J. Rodeheffer, C. G. A. McGregor, S. P. Thomson, and R. J. Gibbons, "Predictive power of the relative lymphocyte concentration in patients with advanced heart failure," Circulation, vol. 97, no. 1, pp. 19-22, 1998.

[35] S. Zhang, J. Diao, C. Qi et al., "Predictive value of neutrophil to lymphocyte ratio in patients with acute ST segment elevation myocardial infarction after percutaneous coronary intervention: a meta-analysis," BMC Cardiovascular Disorders, vol. 18, no. 1, p. 75, 2018.

[36] R. B. Panwar, R. Gupta, B. K. Gupta et al., "Atherothrombotic risk factors \& premature coronary heart disease in India: a case-control study," Indian Journal of Medical Research, vol. 134, no. 1, pp. 26-32, 2011.

[37] P. Seizer and A. E. May, "Therapeutic potential and strategies against leukocyte-platelet interaction in atherosclerosis," Current Vascular Pharmacology, vol. 10, no. 5, pp. 550-554, 2012.

[38] L. Totani and V. Evangelista, "Platelet-leukocyte interactions in cardiovascular disease and beyond," Arteriosclerosis, Thrombosis, and Vascular Biology, vol. 30, no. 12, pp. 23572361, 2010.

[39] E. Nikolsky, C. L. Grines, D. A. Cox et al., "Impact of baseline platelet count in patients undergoing primary percutaneous coronary intervention in acute myocardial infarction (from the CADILLAC trial)," The American Journal of Cardiology, vol. 99, no. 8, pp. 1055-1061, 2007.

[40] M. Gawaz, "Role of platelets in coronary thrombosis and reperfusion of ischemic myocardium," Cardiovascular Research, vol. 61, no. 3, pp. 498-511, 2004.

[41] Y. X. Qian, K. S. Dai, L. L. Zhao, and X. J. Yang, "Effects of remote ischemic post-conditioning on platelet activation of AMI patients," Experimental and Therapeutic Medicine, vol. 16, no. 2, pp. 1273-1277, 2018.

[42] M. Vaduganathan, S. J. Greene, J. Butler et al., "The immunological axis in heart failure: importance of the leukocyte differential," Heart Failure Reviews, vol. 18, no. 6, pp. 835-845, 2013.
[43] A. M. Arruda-Olson, G. S. Reeder, M. R. Bell, S. A. Weston, and V. L. Roger, "Neutrophilia predicts death and heart failure after myocardial infarction," Circulation: Cardiovascular Quality and Outcomes, vol. 2, no. 6, pp. 656-662, 2009.

[44] A. Blum, S. Sclarovsky, E. Rehavia, and B. Shohat, "Levels of T-lymphocyte subpopulations, interleukin- $\beta$, and soluble interleukin-2 receptor in acute myocardial infarction," American Heart Journal, vol. 127, no. 5, pp. 1226-1230, 1994.

[45] A. Shiyovich, H. Gilutz, and Y. Plakht, "White blood cell subtypes are associated with a greater long-term risk of death after acute myocardial infarction," Texas Heart Institute Journal, vol. 44, no. 3, pp. 176-188, 2017.

[46] M. Onsrud, "Influence of in vivo hydrocortisone on some human blood leucocyte sub-populations. II. effects on T cellmonocyte cooperation," Acta Pathologica et Microbiologica Scandinavica, vol. 89, no. 5, pp. 321-327, 1981.

[47] S. Gupta, A. Agrawal, S. Agrawal, H. Su, and S. Gollapudi, “A paradox of immunodeficiency and inflammation in human aging: lessons learned from apoptosis," Immunity \& Ageing, vol. 3, no. 1, p. 5, 2006 .

[48] A. Burlacu, G. Tinica, B. Artene, P. Simion, D. Savuc, and A. Covic, "Peculiarities and consequences of different angiographic patterns of STEMI patients receiving coronary angiography only: data from a large primary PCI registry," Emergency Medicine International, vol. 2020, Article ID 9839281, 7 pages, 2020.

[49] L. Vicent, J. Velásquez-Rodríguez, M. J. Valero-Masa et al., "Predictors of high killip class after ST segment elevation myocardial infarction in the era of primary reperfusion," International Journal of Cardiology, vol. 248, pp. 46-50, 2017. 